\title{
Study on evaluation method of dynamic performance of generator
}

\author{
Xue Min ${ }^{1, a}$, Zhang Wei ${ }^{2, b}$, Li Huiyong ${ }^{1, c}$, Yang Qi ${ }^{1, d}$, Zheng Yongyi $i^{3, e}$ \\ ${ }^{1}$ State Grid Shanxi Electric Power Research Institute, Taiyuan 030001, China \\ ${ }^{2}$ State Grid Information \& Telecommunication CO, of SXPC, Taiyuan 030001, China \\ ${ }^{3}$ State Grid Metering Center of SXPC, Taiyuan 030001, China \\ amig009@126.com, bzhangwei@sx.sgcc.com.cn, 'lihuiyong@sx.sgcc.com.cn, \\ gqi@ sx.sgcc.com.cn,zhengyongyi@ sx.sgcc.com.cn
}

Keywords: dynamic performance; power system; generator;simulation

Abstract. The stability of power system is affected by the dynamic performance of generator. This paper describes a evaluation method of dynamic performance of generator and the establishes the model of dynamic simulation in BPA,which proves that the method is practical and effective by compared with the actual test curve.

\section{Introduction}

With the rapid expansion of ultra high voltage grid, power system of China realizes the leap frog development.Analysis and simulation will provide a powerful technical support for the power system.The model parameters of generator,excitation system,governor and load are important for dynamic simulation of power system,the accuracy of which will directly affect the results of simulation.This paper focuses on the evaluation method of dynamic performance of generator.

\section{The introduction of governor system}

The role of turbine governor system can make the output power equal to the load,which has significant effect on dynamic stability and long-term stability of power system.Using the different model and parameters of turbine governor system in stability calculation of power system, the results of simulation will have a greater difference.The typical model of turbine governor system is shown in figure 1.

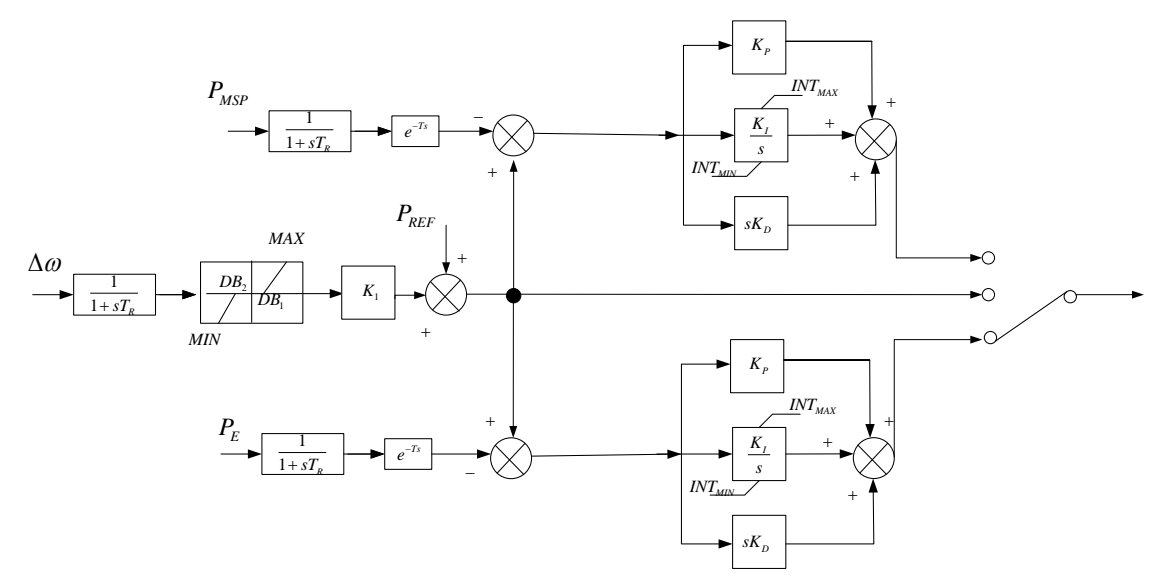

Fig.1 The typical model of turbine governor system

\section{The evaluation indicators of dynamic performance of generator}

The actions of the governor system will be recorded When the system disturbance occurs, which can be used to evaluate the dynamic performance of governor.The load adjustment will take sometime, which is caused by action of Primary frequency, therefore the dynamic performance of governor needs to be evaluated when the load is stable. 
The speed deviation is:

$\Delta n^{\prime}=\frac{\Delta n}{3000}$

Per-unit value of regulating pressure is:

$P_{1 e}=P_{10} \times \frac{\mathrm{P}_{\mathrm{N}}}{N_{0}}$

Per-unit value of the deviation of regulating pressure is:

$\Delta P_{1}^{\prime}=\frac{\Delta P_{1}}{P_{1 e}}$

Per-unit value of load deviation is:

$\Delta N^{\prime}=\frac{\Delta N}{P_{N}}$

Speed fluctuation rate(Considering pressure) is:

$\delta^{\prime}=\frac{\Delta n^{\prime}}{\Delta P_{1}^{\prime}}$

Speed fluctuation rate(Considering load) is:

$\delta^{\prime}=\frac{\Delta n^{\prime}}{\Delta N^{\prime}}$

Frequency variation is:

$f_{\text {MAX }}=\operatorname{Max}\left(f_{i}\right),($ EffetTime $<i<$ UneffectTime $)$

Active power before the disturbance is:

$\bar{N}_{P}=\frac{f_{s}}{2} \sum_{i=a}^{b} N_{i},\left(a=\right.$ StartTime $-\frac{2}{f_{s}}, b=$ StartTime -1$)$

\section{The example of verification}

This paper establishes one machine-infinite bus system in the simulation system of BPA,which is shown in figure 2.

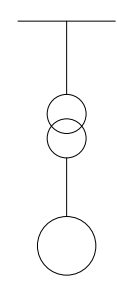

Fig.2 the one machine-infinite bus system

The model of turbine governor system established in this paper adopts experimental data,using the actual frequency difference in experiment as the input.The dynamic performance of turbine governor system can be evaluated by comparing the result of simulation and experiment.

The comparison of simulation frequency difference with experimental frequency difference is shown in figure 3. 


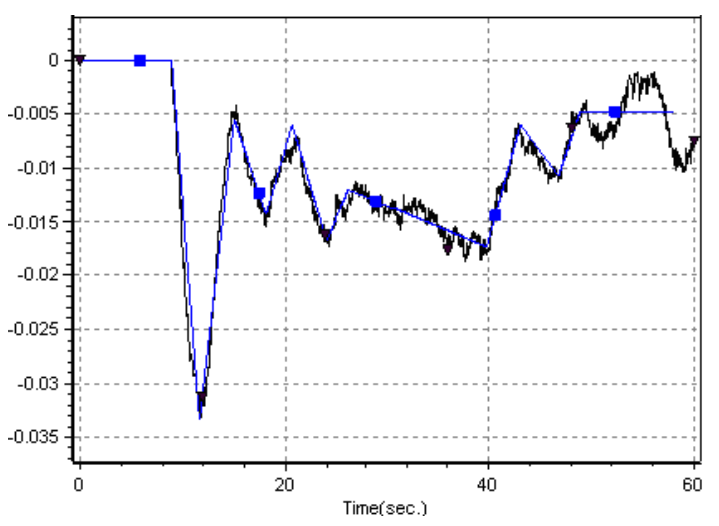

Fig.3 The comparison of simulation with experimental frequency difference

The step-up comparison of simulation active power with experimental active power in CCS power control mode is shown in figure 4.

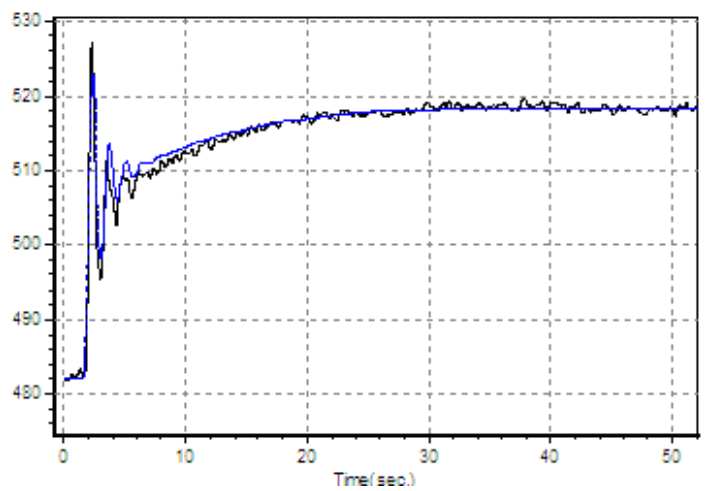

Fig.4 The step-up comparison of simulation with experimental active power

The step-down comparison of simulation active power with experimental active power in CCS power control mode is shown in figure 5.

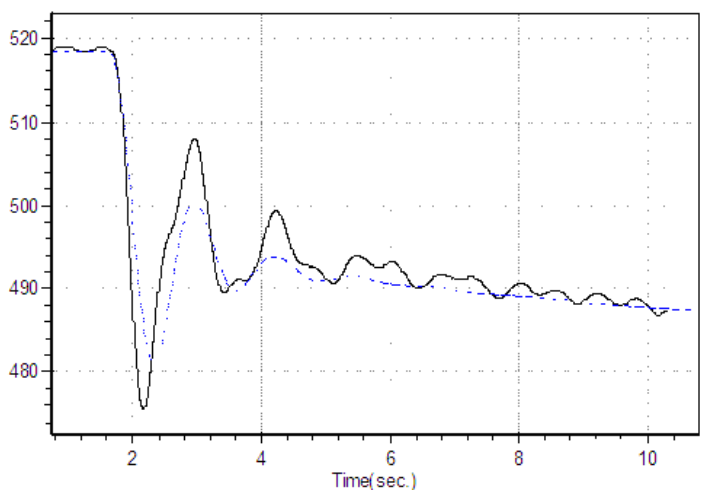

Fig.5 The step-down comparison of simulation with experimental active power

\section{Conclusions}

This paper establishes one machine-infinite bus system in the simulation system of BPA, using the experimental parameters of turbine governor system to simulate, and comparing the simulation results with the field test results. The study of this paper shows that the simulation curves are more fit to experimental curves, which illustrate the evaluation method proposed in this paper is practical and effective.

\section{References}

[1] Zhu Fang,Tang Yong,Zhang Dongxia,et al. Influence of Excitation and Governor Model Parameters on Simulation of Large-Disturbance Test in Northeast China Power Grid[J].Power System Technology,2007,31(4):69-74. 
[2] Yue Chengyan,Tian Fang,Zhou Xiaoxin,et al.Application of hybrid simulation of power system electromagnetic-electromechanical transient process[J].Power System Technology,2006,30(11):1-5.

[3] Chang Hui,Liu wenying,Xing Zhou,et al.Online application of power system transient stability computation[J].Power System Technology,2007,31(13):54-58.

[4] Tang Yong, Zhu Fang, Zhang Dongxia,et al. Commissioning tests of interconnection of north china power grid with northeast china power grid[J].Power System Technology,2001,25(11):46-49.

[5] Li Yalou,Zhou Xiaoxin,Wu Zhongxi.Personal computer cluster based parallel algorithms for power system electromechanical transient stability simulation[J].Power System Technology, 2003,27 (11):6-12.

[6] Wu Cheng,Xu Zheng,Pan WuLue,et al.New Developments on Turbine-governor Modeling and Parameter Identification [J]. Power System Technology,2007,31(1):179-182.

[7] Meng Anbo,Yin Hao.Multi-Agent genetic algorithms for PID governor[J].Electric Power Automatic Equipment,2012,32(9):128-133.

[8] Cao Chengjie,Mo Yueping.Research of self-adaptive PID hydraulic turbine governor based on modern intelligence control technology[J].Power System Protection and Control,2010, 38(3):81-85. 